\title{
RF Characteristics of Open Stubs on PES Substrate for Application to Capacitive Matching Components on Flexible MMIC
}

\author{
Young Yun ${ }^{\dagger}$ and Jang-Hyeon Jeong \\ Department of Radio Communication and Engineering, Korea Maritime and Ocean University, Busan 606-791, Korea \\ Hong-Seung Kim \\ Department of Nano Semiconductor Engineering, Korea Maritime and Ocean University, Busan 606-791, Korea \\ Nak-Won Jang \\ Department of Electrical and Electronics Engineering, Korea Maritime and Ocean University, Busan 606-791, Korea
}

Received October 7, 2014; Revised October 27, 2014; Accepted December 11, 2014

\begin{abstract}
In this work, open stubs were fabricated on a polyether sulfone (PES) substrate, and their basic radio frequency (RF) characteristics were investigated for application to RF matching components of a flexible monolithic microwave integrated circuit (MMIC). According to the results, an open stub employing coplanar waveguide (OSCPW) on PES exhibited much lower loss than that on silicon substrate. The OSCPW with a length of $500 \mu \mathrm{m}$ on PES showed capacitance values of $0.031 \sim 0.044 \mathrm{pF}$ from 0.5 to $50 \mathrm{GHz}$. For application to a relatively high-value capacitive matching, an open stub employing a fishbone-type transmission line (OSFTTL) was fabricated on PES, and its characteristics were investigated. The OSFTTL showed much higher capacitance values than the OSCPW due to the high effective permittivity value. Specifically, the OSFTTL on PES showed capacitance values of $0.066 \sim 0.24 \mathrm{pF}$ from 0.5 to 50 $\mathrm{GHz}$, which are higher than those for the open stub on silicon substrate. The above results indicate that the OSCPW and OSFTTL on PES can be effectively used for application to low/high-value capacitive matching components on microwave and millimeter wave flexible MMIC. To the best of the authors' knowledge, this work is the first report of the investigation of RF capacitive matching components on PES substrate.
\end{abstract}

Keywords: Open stub, Polyether sulfone (PES), Flexible monolithic microwave integrated circuit (MMIC), Open stub employing coplanar waveguide (OSCPW), Open stub employing fishbone-type transmission line (OSFTTL)

\section{INTRODUCTION}

Flexible electronics have attracted significant attention due to their vast potential applications in a variety of areas such as

\footnotetext{
${ }^{\dagger}$ Author to whom all correspondence should be addressed: E-mail: yunyoung@kmou.ac.kr
}

Copyright (C2015 KIEEME. All rights reserved.

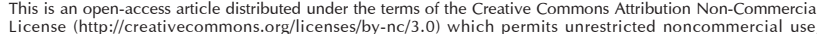
License (http:///ceativecommons.org/licenses//hy-nc/3.0) which permits unrestricted
distribution, and reproduction in any medium, provided the orignal work is properly cited flexible display, smart tag, and wearable electronics [1]. Especially, for transparent flexible displays optimized for mobile communication devices, the integration of RF components on a transparent flexible substrate is highly desirable. Recently, the PES substrate has attracted much attention for the transparent flexible display application due to its excellent heat-resistance, high transparency, and good flexibility [2,3].

For this reason, the PES has been employed for application to the flexible monolithic microwave integrated circuit (MMIC) [4]. Especially, transmission lines have been realized on PES, and their RF characteristics have been studied thoroughly. However, 
the study on the integration of RF devices on a flexible PES substrate is still in its early stage. Therefore, impedance matching components such as the capacitor has not yet been studied thoroughly. For the realization of a flexible MMIC, passive matching elements such as open stubs should be intensively studied, because they are essential components in millimeter wave as well as microwave frequency. In this work, we fabricated open stubs on a PES substrate, and studied their basic RF characteristics for application to RF matching components on flexible MMIC.

\section{RF CHARACTERISTICS OF OPEN STUB EMPLOYING COPLANAR WAVEGUIDE ON PES}

First, an open stub employing a coplanar waveguide (OSCPW) was fabricated on PES and its return loss was measured.

Figures 1 and 2 show the open stub on PES, and its return loss from DC to $50 \mathrm{GHz}$, respectively. For fabrication of the OSCPW on PES, Au/Ti was deposited on a PES substrate with a thickness of $200 \mu \mathrm{m}$, and the thickness of the Au/Ti was $2 \mu \mathrm{m}$. The width and length of the OSCPW are 70 and $500 \mu \mathrm{m}$, respectively. For a comparison, OSCPW with the same size was fabricated on a silicon substrate, and its return loss was also plotted in Fig. 2, because silicon substrate is the most commonly used semiconducting substrate for commercial RF applications. In the case of an ideal lossless open stub, its return loss $\left(\mathrm{S}_{11}\right)$ moves from open point clockwise along the outermost circle as frequency increases, because the absolute value of its return loss is 1 in all frequencies. As shown in Fig. 2, the trace of the return loss of the OSCPW on PES is very similar to an ideal lossless open stub. On the other hand, as frequency increases, the return loss of the OSCPW on the silicon substrate moves inwards, deviating from the outermost due to attenuation from a loss of silicon substrate. According to our previous results [4], the Q-factor of the coplanar waveguide on the PES substrate was 40.3 at $46.7 \mathrm{GHz}$, which was much higher than that on the silicon substrate $[5,6]$ and comparable to that on the GaAs substrate [7]. The above results indicate that the open stub matching circuit on a PES substrate can be employed for application to millimeter wave as well as microwave due to its very low loss.

The open stub is used as a shunt capacitor on an RF circuit. Therefore, the capacitance of the OSCPW on PES was extracted from the measured return loss. Firstly, the open stub was expressed by an equivalent RC circuit, which is shown in Fig. 3.

In this figure, R corresponds to the resistance originating from the loss of open stub. As is well known, the relation between input impedance $Z_{\text {in }}$ and return loss $S_{11}$ of the open stub can be expressed as follows [8],

$$
Z_{\text {in }}=Z_{0} \frac{1+S_{11}}{1-S_{11}}
$$

The above input impedance is the same as that calculated from the equivalent circuit shown in Fig. 4. Therefore, the capacitance of the open stub can be extracted from the following equations.

$$
Z_{\text {in }}=R+\frac{1}{j \omega C}=R-j \frac{1}{\omega C}=Z_{0} \frac{1+S_{11}}{1-S_{11}}
$$

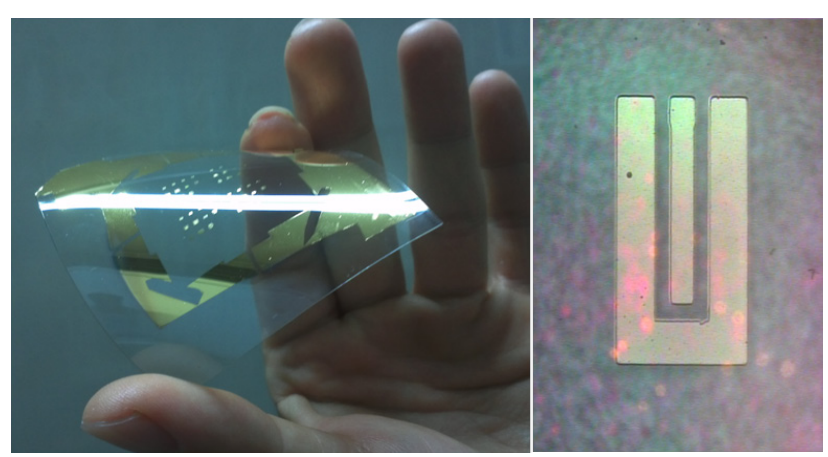

Fig. 1. Photograph of the open stub employing coplanar waveguide (OSCPW) on PES.

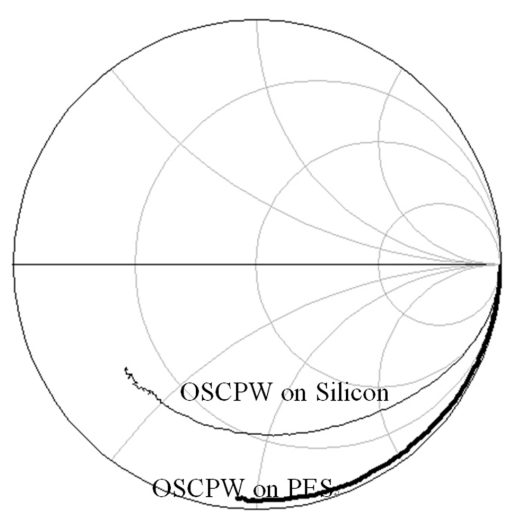

Fig. 2. Measured return loss $\left(\mathrm{S}_{11}\right)$ of the OSCPWs on PES and silicon substrate.

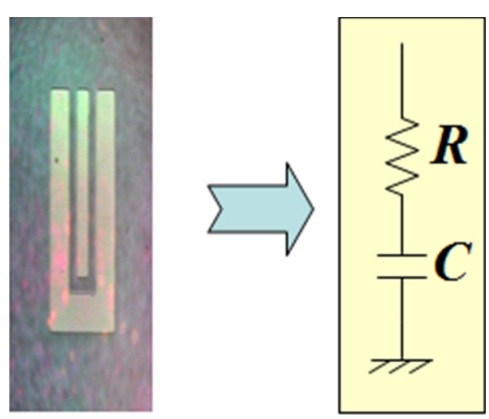

Fig. 3. Equivalent circuit of the OSCPW on PES.

$$
-\frac{1}{\omega C}=i m\left(Z_{0} \frac{1+S_{11}}{1-S_{11}}\right)
$$

Where $\omega$ is the angular frequency and im (z) refers to an imaginary part of $\mathrm{z}$.

Figure 4 shows the capacitance of the OSCPW on PES. For a comparison, the capacitance of the OSCPW on the silicon substrate was also plotted. As shown in this figure, the OSCPW on PES exhibits capacitance values lower than those on the silicon substrate. In particular, the OSCPW on PES shows capacitance values of $0.031 \sim 0.044 \mathrm{pF}$ from 0.5 to $50 \mathrm{GHz}$, while that on silicon substrate shows capacitance values of $0.066 \sim 0.12 \mathrm{pF}$ in the same frequency range. The above result can be explained from the following equation. The capacitance of the open stub can be 


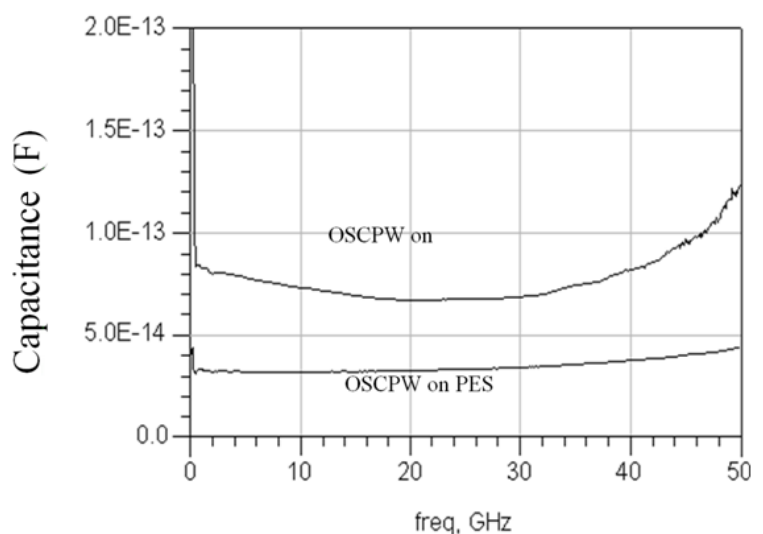

Fig. 4. Measured capacitance values of the OSCPW on PES and silicon substrate.

given by [8], where $\beta$ and 1 are the propagation constant of the transmission line and length of the open stub, respectively, and $\mu$ and $\varepsilon_{\text {eff }}$ are the permeability and effective permittivity of the transmission line, respectively. From the above equation, the capacitance of the open stub is proportional to $\left(\varepsilon_{\mathrm{eff}}\right)^{0.5}$.

According to our results, the coplanar waveguide on PES showed $\varepsilon_{\text {eff }}$ of 2.35 2.9 from 10 to $50 \mathrm{GHz}$, while the coplanar waveguide on silicon substrate showed $\varepsilon_{\text {eff }}$ of $5.9 \sim 13.6$ from 10 to $50 \mathrm{GHz}$ [4]. Therefore, the OSCPW on PES exhibits capacitance values lower than that on the silicon substrate due to the lower effective permittivity of the PES substrate.

$$
C=\frac{\tan (\beta l)}{\omega Z_{0}}=\frac{\tan (\omega \sqrt{\mu \varepsilon} l)}{\omega Z_{0}}=\frac{\tan \left(\omega \sqrt{\mu \varepsilon_{0} \varepsilon_{e f f}} l\right)}{\omega Z_{0}}
$$

\section{RF CHARACTERISTICS OF OPEN STUB EMPLOYING FISHBONE-TYPE TRANSMISSION LINE ON PES}

Sometimes, a high capacitance value is needed for impedance matching in the microwave frequency range, and requires a high capacitive open stub. From Eq. (4), we can see that the effective permittivity of the transmission line should be increased in order to enhance the capacitance value of the open stub.

According to transmission line theory, the equivalent periodic capacitance and inductance of the transmission line are proportional to the effective permittivity, which can be explained from the following equations [8].

$$
\begin{aligned}
& \beta=\omega \sqrt{\mu \varepsilon}=\omega \sqrt{\mu \varepsilon_{0} \varepsilon_{e f f}}=\omega \sqrt{L C} \\
& \varepsilon_{\text {eff }}=\frac{L C}{\mu \varepsilon_{0}}
\end{aligned}
$$

where $\mathrm{L}$ and $\mathrm{C}$ are the periodic equivalent inductance and capacitance of the transmission line, respectively. According to our previous results [4], the fishbone-type transmission line (FTTL)
OSFTTL

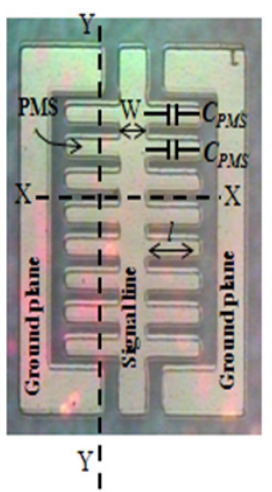

(A cross-sectional view of $\mathrm{X}$-X direction)

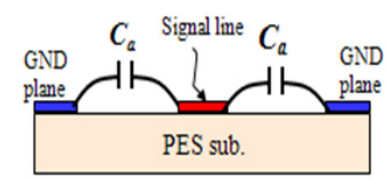

(Periodical inductance and capacitance for FTTL Structure)

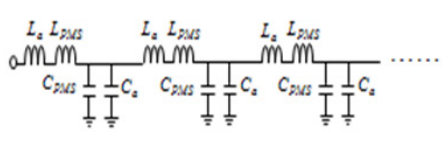

Fig. 5. Structure of the open stub employing fishbone-type transmission line (OSFTTL) on PES.

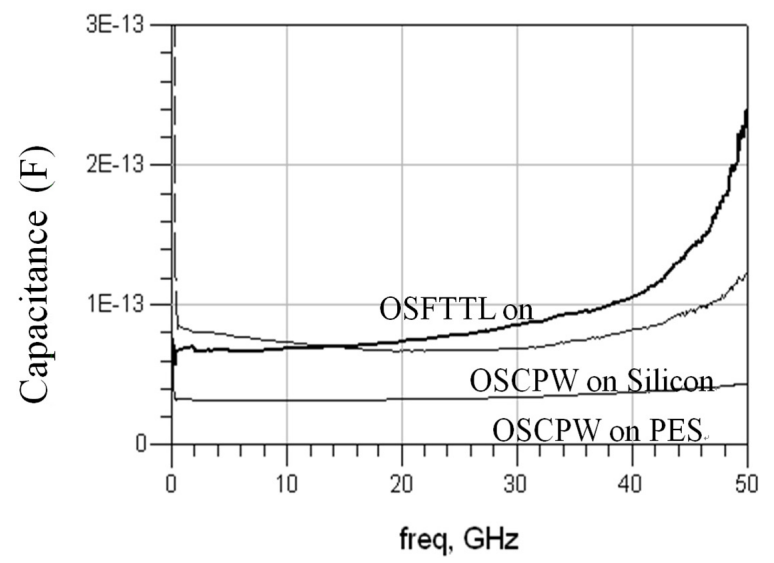

Fig. 6. Measured capacitance values of the OSFTTL and OPCPW on PES.

on PES showed a much higher periodic capacitance and inductance than the conventional coplanar waveguide on PES. Due to an enhancement of the periodic capacitance and inductance value, the FTTL on PES showed a higher $\varepsilon_{\text {eff }}$ than the conventional coplanar waveguide on PES.

Especially, the FTTL showed a $\varepsilon_{\text {eff }}$ of $7.2 \sim 9.5$ from 5 to $50 \mathrm{GHz}$, while the coplanar waveguide on PES showed the $\varepsilon_{\text {eff }}$ of $2.45 \sim$ 2.97 in the same frequency range [4]. In addition, FTTL showed a higher $\varepsilon_{\text {eff }}$ than the coplanar waveguide on the silicon substrate at a higher frequency range than $10 \mathrm{GHz}$.

In this work, using the FTTL [4], we fabricated an open stub for application to a relatively high-value capacitive matching component on a flexible MMIC. Specifically, we fabricated an open stub employing the fishbone-type transmission line (OSFTTL), and designed the appropriate structure of the open stub in order to enhance the effective permittivity by increasing the periodic capacitance and inductance. Figure 5 shows an open stub employing a fishbone-type transmission line (OSFTTL) on PES. As shown in this figure, the OSFTTL consists of a fishbonetype center line and ground planes. The fishbone-type center line consists of a signal line and periodic metal strips (PMS's).

The OSCPW employing a conventional coplanar waveguide has only a periodical capacitance $\mathrm{Ca}$ between the line and ground plane, while the OSFTTL has an additional periodical shunt capacitance CPMS as well as Ca, because each PMS operates as a periodic open stub capacitor in operating frequency. In 
addition, the OSCPW has only a periodical inductance $\mathrm{L}_{\mathrm{a}}$ due to the current flowing across the signal line, while the OSFTTL has additional inductance $\mathrm{L}_{\mathrm{PMS}}$ as well as $\mathrm{L}_{\mathrm{a}}$ due to the current flowing across the PMS.

The capacitance values of the OSFTTL are shown on the PES. For a comparison, the capacitance of the OSCPW on the PES and silicon substrate was also plotted. As shown in this figure, the OSFTTL exhibits much higher capacitance values than the OSCPW on PES. In particular, the OSFTTL on PES shows capacitance values of $0.066 \sim 0.24 \mathrm{pF}$ from 0.5 to $50 \mathrm{GHz}$, while the OSCPW on PES shows capacitance values of $0.031 \sim 0.044 \mathrm{pF}$ from 0.5 to 50 $\mathrm{GHz}$. In addition, in a frequency range higher than $14 \mathrm{GHz}$, the OSFTTL on PES shows capacitance values higher than OSCPW on silicon substrate. The above results indicate that the OSFTTL structure on PES can be effectively used as a shunt capacitor for a flexible RF circuit that requires a relatively high-value capacitive matching component.

\section{CONCLUSIONS}

In this work, open stubs were fabricated on a PES substrate, and their basic RF characteristics were thoroughly studied for application to RF capacitive matching components on flexible MMIC. According to the results, the trace of the return loss of the OSCPW on PES was very similar to an ideal lossless open stub, and it showed a loss much lower than the OSCPW on silicon substrate. The OSCPW with a length of $500 \mu \mathrm{m}$ on PES showed capacitance values of $0.031 \sim 0.044 \mathrm{pF}$ from 0.5 to $50 \mathrm{GHz}$, which is suitable for application to a low-value shunt capacitive matching component in the millimeter wave frequency range. For application to a relatively high-value capacitive matching, a properly designed OSFTTL was proposed. The OSFTTL showed much higher capacitance values than the OSCPW due to high effective permittivity $\varepsilon_{\text {eff }}$ value. Concretely, the OSFTTL on PES showed capacitance values of $0.066 \sim 0.24 \mathrm{pF}$ from 0.5 to 50 $\mathrm{GHz}$. In addition, in a frequency range higher than $14 \mathrm{GHz}$, the
OSFTTL on PES showed capacitance values higher than OSCPW on silicon substrate. The above results indicate that the OSCPW and OSFTTL on PES can be effectively used for application to low/high-value capacitive matching components on microwave and millimeter wave flexible MMIC. To the best of the authors' knowledge, this work is the first report of the investigation of RF capacitive matching components on a PES substrate.

\section{ACKNOWLEDGMENTS}

This research was financially supported by the LG Yonam Foundation. This work was also supported by a National Research Foundation of Korea (NRF) grant funded by the Korea government (MSIP) (2014R1A2A1A11049844).

\section{REFERENCES}

[1] Y. Sun, and J. A. Rogers, Advanced Materials, 19, 1987 (2007).

[2] E. Celik, H. Park, H. Choi, and H. Choi, Water Research, 45, 274 (2011). [DOI: http://http://dx.doi.org/10.1016/ j.watres.2010.07.060].

[3] R. Rajasekaran, M. Alagar, and C. K. Chozhan, Express Polymer Letter, 2, 339 (2008). [DOI: http://http://dx.doi.org/10.3144/ expresspolymlett.2008.40].

[4] Y. Yun, H. S. Kim, and N. W. Jang, ETRI J., 36, 106 (2014). [DOI: http://http://dx.doi.org/10.4218/etrij.14.0113.0124].

[5] J. R. Long, IEICE Trans. Electron., E86-C, 1022 (2003).

[6] C. H. Doan, S. Emami, A. M. Niknejad, and R. W. Broderson, Proc. of IEEE International Solid-State Circuits Conference, Session 24.4 (2004).

[7] W. H. Haydl, T. Kitazawa, J. Braunstein, R. Bosch, and M. Schlechtweg, IEEE MTT-S International Microwave Symposium Digest, 2, 691 (1991).

[8] D. M. Pozar, Microwave Engineering (Reading, MA, AddisonWesley, 1990). 